\title{
Types and frequency of preanalytical mistakes in the first Thai ISO 9002: 1994 certified clinical laboratory, a 6 - month monitoring Viroj Wiwanitkit
}

Address: Department of Laboratory Medicine, Faculty of Medicine, Chulalongkorn University, Bangkok, Thailand

E-mail: wviroj@pioneer.netserv.chula.ac.th

Published: 16 October 2001

BMC Clinical Pathology 200I, I:5
Received: 26 May 200

Accepted: 16 October 2001

This article is available from: http://www.biomedcentral.com/1472-6890/I/5

(C) 200I Wiwanitkit; licensee BioMed Central Ltd. Verbatim copying and redistribution of this article are permitted in any medium for any non-commercial purpose, provided this notice is preserved along with the article's original URL. For commercial use, contact info@biomedcentral.com

\begin{abstract}
Background: Reliability cannot be achieved in a clinical laboratory through the control of accuracy in the analytical phase of the testing process alone. Indeed a "mistake" can be defined as any defect occuring during the testing process. In the analysis of clinical specimens, there are many possible preanalytical sources of error. Therefore, the application of quality system to laboratory testing requires total quality management throughout the laboratory process, including the preanalytical and postanalytical phases. ISO 9002:1994 is a model for quality assurance in production, installation, and servicing, which includes a number of clauses providing guidance for implementation in clinical laboratories. Our laboratory at King Chulalongkorn Memorial Hospital, the largest Thai Red Cross Society hospital, is the first clinical laboratory in Thailand with ISO 9002:1994 certified for the whole unit.
\end{abstract}

Method: In this study, we evaluated the frequency and types of preanalytical mistakes found in our laboratory, by monitoring specimens requested for laboratory analyses from both in-patient and out-patient divisions for 6 months.

Result: Among a total of 935,896 specimens for 941,902 analyses, I,048 findings were confirmed as preanalytical mistakes; this was a relative frequency of 0.1 I \% $(I, 048 / 935,896)$. A total of I,240 mistakes were identified during the study period. Comparing the preanalytical mistakes to other mistakes in the laboratory process monitored in the same setting and period, the distribution of mistakes was: preanalytical $84.52 \%$ (I,048 mistakes), analytical $4.35 \%$ (54 mistakes), and postanalytical II.I3\% (I38 mistakes). Of I,048 preanalytical mistakes, 998 (95.2\%) originated in the care units. All preanalytical mistakes, except for 12 (I.15\%) relating to the laboratory barcode reading machine, were due to human error.

Conclusion: Most mistakes occurred before samples were analysed, either during sampling or preparation for analysis. This suggests that co-operation with clinicians and personnel outside the laboratory is still the key to improvement of laboratory quality.

\section{Introduction}

Quality is the heart in management of all laboratories. Due to the good governance concepts, accountability of the whole laboratory process is the main focus of current concern in laboratory medicine. Due to the laboratory quality cycle, reliability cannot be achieved in a clinical laboratory through the control of accuracy in the analytical phase of testing process alone. There should be a certification on the whole laboratory, but not on single analytical process. Precision and accuracy of analyses are 
not only determined by the analytical procedure but also by preanalytical factors, such as contamination and loss during sampling and sample preparation. Under the broad umbrella of the preanalytical phase can be included requesting test, specimen collection, handling and processing in the process before complete distribution of test samples to multiple work stations [1,2]. In the total analytical process of clinical specimens, there are many possible preanalytical sources of error [3].

According to a recent study, mistakes in the preanalytical phase were upto $50 \%$ [4]. Therefore, the application of quality system to laboratory testing requires total quality management in the whole laboratory process, including preanalytical and postanalytical phases. The major aim of the quality system is to reduce or, ideally, eliminate all defects within the whole laboratory process.

ISO 9002:1994 [5], a quality system in ISO 9000 series, is a model for quality assurance in production, installation, and servicing, which includes a number of clauses providing guidance for the implementation of the quality system. This quality system starts from general quality and documentation plans followed by the internal audit plan step and the launch of the quality system. Finally, the assessment by an external team from an accredited third-party organization and final certification for compliance to ISO 9002 must be performed. The main topics according to ISO 9002 standard include management responsibility, organization, quality system, control of nonconforming product, statistical techniques and etc. Like other manufacturing processes, the clinical laboratory process is a type of service therefore the ISO 9002 can be applied $[5,6]$. As the realization of the ISO 9002 standard in the laboratory, all already mentioned topics can also be used [7]. This quality system, which must be maintained and undergo external audit at regular intervals so that certification is confirmed, ensures the product (laboratory analysis service) to clinicians conform regularly to predefined levels of quality [6].

In Thailand, the ISO 9002:1994 quality system is also implemented for the clinical laboratory. Our laboratory at King Chulalongkorn Memorial Hospital, the largest Thai Red Cross Society hospital, is the first clinical laboratory in Thailand with ISO 9002:1994 certified for the whole unit, including structures, processes and so on. Monitoring of the preanalytical mistakes is one commitment in our quality plan. The aim of this study was to evaluate the types and frequency of mistakes in our laboratory in the preanalytical testing process. We gave the operative definition for a "mistake" as any defect during the entire process, that influenced in any way the quality of the laboratory service.

\section{Materials and methods The setting}

The Department of Laboratory Medicine of the University-Hospital of Chulalongkorn, is a large laboratory service providing tests for clinical chemistry, hematology and coagulation. The laboratory serves King Chulalongkorn Memorial Hospital, the largest Thai Red Cross Society hospital, with 2900 beds and highly specialized care units. The laboratory department performs about two million tests per year with individual spaces, instruments, and staff. Each instrument is connected to the laboratory information system, $<5 \%$ of the results being manually entered into the computer.

Considering the laboratory workflow, the service of the laboratory starts from the receptions of requests, specimens and request forms, to distribution of the laboratory results to the patients (for Out-Patient Division) and clinical wards (for In-Patient Division). The laboratory consists of 7 units as request reception unit, hematology unit, clinical chemistry unit, clinical microscopic unit, special test unit, quality control unit and laboratory result distribution unit. The ISO 9002:1994 has been used as the quality system of the laboratory and certified for the whole since year 2000. The laboratory quality committee, consisted of the hospital administrators, laboratory department administrator and the heads of medical technologists from all units of the laboratory was also set. According to the documentation plans, we produced of (i) one quality manual, which provided a summary on how the laboratory operates with a quality system in compliance with the ISO 9002 clauses, (ii) 10 quality system procedures, which provided more details on the activities, (iii) 71 work instructions, which provided stepwise descriptions of all activities, and (iv) 82 forms for data collection and storage (Table 1).

Table I: Examples of documents according to ISO 9002:1994 in our setting.

\begin{tabular}{ll}
\hline \multicolumn{1}{c}{ types } & \multicolumn{1}{c}{ examples } \\
\hline I. Quality system procedure & $\begin{array}{l}\text { Statistical technique } \\
\text { Process control } \\
\text { Management review } \\
\text { Fire prevention } \\
\text { 2. Work instruction }\end{array}$ \\
3. Form & $\begin{array}{l}\text { Datient education } \\
\text { mistake } \\
\text { Client satisfaction survey form }\end{array}$ \\
\hline
\end{tabular}




\section{Procedure of data collection}

Based on the recommendations for ISO 9002 system for the hospital by the Technology Promotion Association (Thailand - Japan) [7], the laboratory quality committee decided that the rate of preanalytical mistakes is a quality indicator of our laboratory. The others quality indicators were the client satisfaction, complaint, turnaround time and etc. According to the documentation plans, the specific quality system procedure, which provided the details on monitoring of preanalytical mistakes as a quality indicator according to the statistical technique prescribed by the ISO 9002 standard, was set [7]. As previously described, the definition of the "mistake in the preanalytical phase" is any defect during the preanalytical phase, including all processes before complete distribution of test samples to multiple work stations, that influenced in failure of further laboratory process. These preanalytical mistakes include physician's order missed, patient misidentification, specimen collected in insufficient quantity, inappropriate container used, inappropriate quality of specimen, specimen lost in transit and etc [8].

Furthermore, specific work instruction, which provided stepwise descriptions of the activities, and the specific data record forms for data collection and storage were also set. Briefly, all medical technologists in all units of the laboratory were asked to pay maximal critical attention to all received requests. These personnel were provided with a special notebook in which any "suspect" sample was recorded, together with all pertinent information. Then consultation to the head of medical tech- nologist of the unit was done. The head of medical technologist rechecked and reviewed all reported cases before making final decision. In cases that the preanalytical mistakes were made from final decisions, they were recorded into the specific record form (Table 2). Data record forms from all units were collected monthly and transferred to the laboratory quality committee for further statistical analysis. After review of the monthly data, the laboratory quality committee will set the corrective strategy for the identified problems.

\section{Statistical analysis}

In this study, recorded data about preanalytical mistakes of the requests from both In-Patient and Out-Patient Divisions of the hospital were selected, and all laboratory data for these departments were also strictly monitored for 6 months (October 2000 to March 2001). The sums of mistakes were calculated. Their relative frequencies comparing to the total specimens were also calculated and presented as percentage. The statistical significance level accepted in this study was set at $\mathrm{P}$ value equal to 0.05. The difference between relative frequencies of mistakes observed in the departments considered was tested by proportional $\mathrm{Z}$ test [9].

\section{Results}

Among a total of 935,896 specimens for 941,902 analyses, 1,048 findings were confirmed as preanalytical mistakes; this was a relative frequency of $0.11 \%(1,048 /$ 935,896). In the Out-Patient Division, the relative frequency of mistakes was not significant different (Table 3) from the frequency from the In-Patient Division $(\mathrm{P}>$

Table 2: Example of data record form for preanalytical mistake

\begin{tabular}{lccc}
\hline Date & time & Source of request & $\begin{array}{c}\text { Type* and detail } \\
\text { of identified mistake }\end{array}$ \\
\hline
\end{tabular}

\footnotetext{
* classified as physician's order missed, patient misidentiflcation, specimen collected in insufficient quantity, inappropriate container used, inappropriate quality of specimen, specimen lost in transit and etc.
} 
Table 3: Preanalytical mistakes in laboratory testing.

\begin{tabular}{lccccc}
\hline Division & $\begin{array}{c}\text { Number } \\
\text { of samples* }\end{array}$ & $\begin{array}{c}\text { Number of } \\
\text { requested } \\
\text { tests }\end{array}$ & $\begin{array}{c}\text { Number of } \\
\text { preanalytical } \\
\text { mistakes }\end{array}$ & Frequency (\%) \\
\hline & & & & & \\
In-Patient & 298,769 & 304,906 & 426 & 0.143 & $0.129-0.156$ \\
Out-Patient & 441,268 & 457,013 & 622 & 0.141 & $0.130-0.152$ \\
Total & 935,896 & 941,902 & 1,048 & 0.112 & $0.105-0.119$ \\
& & & & &
\end{tabular}

* The number of requested tests were greater than the number of samples because more than one test could be performed on a sample.

o.80). Considering the preanalytical mistakes, the most common faults (Table 4) depended on inaccurate procedures for sample collection, including inappropriate quality of specimen (47.04\%), inappropriate quantity of specimen (11.55\%), and utilization of an inappropriate container $(0.57 \%)$. Other frequent mistakes were physician's order missing (14.03\%) and wrong identification of the patient (26.81\%), causing a risk of delay; an erroneous diagnosis; and inappropriate treatment. Of 1,048 preanalytical mistakes, 998 (95.2\%) originated in the care units; the laboratory including laboratory reception area, of course, has no control over such defects.

\section{Discussion}

Only a few studies evaluated the frequency and types of mistakes in the preanalytical process. In retrospect, there were totally 1,240 identified mistakes during the study period. Comparing the preanalytical mistakes to other mistakes in the same setting and period, the distribution of mistakes was preanalytical $84.52 \%(1,048$ mistakes), analytical $4.35 \%$ (54 mistakes), and postanalytical $11.13 \%$ (138 mistakes). This result ap-

Table 4: Types of preanalytical mistakes in laboratory testing.

\begin{tabular}{|c|c|c|}
\hline \multirow[t]{2}{*}{ Defects detection steps } & \multicolumn{2}{|c|}{ Defects found } \\
\hline & number & Frequency $(\%)$ \\
\hline $\begin{array}{l}\text { Wrong identification of the } \\
\text { patient }\end{array}$ & 281 & 26.81 \\
\hline Physician's order missed & 147 & 14.03 \\
\hline Inappropriate container used & 6 & 0.57 \\
\hline $\begin{array}{l}\text { Inappropriate quantity of } \\
\text { specimen }\end{array}$ & 121 & 11.55 \\
\hline Inappropriate quality of specimen & 493 & 47.04 \\
\hline
\end{tabular}

pears to confirm the high rate of mistakes in the preanalytical phase, which mentioned in the recent previous studies $[4,10]$.

However, the sum of preanalytical mistakes and their relative frequency are surprisingly high; this may have partly depended on the fact that most preanalytical mistakes originated in the care units, not in the laboratory. Less than $10 \%$, less than the previous report [10], of preanalytical mistakes resulted from the laboratory.

It has been stated that all faults, except for 12 mistakes $(1.15 \%)$ relating to laboratory information system, depend on human mistakes. Considering the mistakes resulted by the laboratory information system, all were due to barcode reading machine errors. This result also confirms the previous study in the Hospitals of Tilburg, which stated that $93-97 \%$ of mistakes in the laboratory process resulted from human error [11].

We also attempted to ascertain the effect of laboratory mistakes. All mistakes were associated with repeat specimen collection for laboratory tests, or repeat laboratory analysis thus resulting in an unjustified increase in costs.

Today, the quality system for clinical laboratories must include promotion of accuracy in the analytical phase as well as the assurance of the reliability of preanalytical and postanalytical activities. Despite using of ISo9002: 1994 quality system in the laboratory, a large percentage of mistakes occurred in the preanalytical phases. This indicates that the active monitoring and feedback control of all potential defects generated by non-laboratory personnel, are essential to enable the inclusion of steps outside the laboratory. Co-operation with clinicians and personnel outside the laboratory is therefore the key to improvement $[12,13]$. In order to reduce the preanalytical mistakes originated in the care units, a regular feedback system (such as distribution of protocol for proper specimen collection) to the clinicians and personnel outside the laboratory is also set in our setting. 


\section{Competing interests}

None declared.

\section{Acknowledgement}

The author has to acknowledge all of the laboratory workers who participated in the system.

\section{References}

I. Irjala KM, Gronroos PE: Preanalytical and analytical factors affecting laboratory results. Ann Med 1998, 30:267-272

2. Narayanan S: The preanalytic phase - An important component of laboratory medicine. Am J Clin Pathol 2000, I I 3:429-452

3. Schmitt $Y$ : Influence of preanalytical factors on the atomic absorption spectrometry determination of trace elements in biological samples. J Trace Elem Electrolytes Health Dis 1987, I:I07II4

4. Boone J, et al: Transfusion medicine monitoring practices. Arch Pathol Lab Med 1995, I 1 9:999-1006

5. Sim P: ISO 9000:2000 revisions and their implications. Med Device Technol 2000, I I:45-47

6. Sirchia G, et al: Implementation of a quality system (ISO 9000 series) for placental blood banking. J Hematother 1998, 7:19-35

7. Pruksaptitikul S: ISO $\mathbf{9 0 0 2}$ system for the hospital. Bangkok: Technology Promotion Association (Thailand - Japan) 1998

8. Young DS, Bermes EW: Specimen collection and processing: source of biological variation. In: Clinical Chemistry. (Edited by Burtis CA, Ashwood ER). Philadelphia, WB Saunders, 199833-52

9. Walpole RE, Myers RH: Probability and Statistics of Engineering and Scientists. New York: McMillan 1972

10. Plebani $M$, Carraro $P$ : Mistakes in a stat laboratory: types and frequency. Clin Chem 1997, 43:1348-135I

II. Goldschmidt HMJ, Lent RW: Gross errors and work flow analysis in the clinical laboratory. Klin Biochem Metab 1995, 3: I 3 I- I 40

12. Plebani M, Chiozza ML: Audit in laboratory medicine. Eur J Clin Chem Clin Biochem 1996, 34:655-657

13. Boon DJ: Evaluating laboratory performance. Arch Pathol Lab Med 1 988, I I 2:354-356

Publish with BioMed Central and every scientist can read your work free of charge

"BioMedcentral will be the most significant development for disseminating the results of biomedical research in our lifetime."

Paul Nurse, Director-General, Imperial Cancer Research Fund

Publish with BMC and your research papers will be:

- available free of charge to the entire biomedical community

- peer reviewed and published immediately upon acceptance

- cited in PubMed and archived on PubMed Central

- yours - you keep the copyright 